\title{
Intelligent Management of On-street Parking Provision for the Autonomous Vehicles Era
}

\author{
Qiming $\mathrm{Ye}^{1}$, Simon M Stebbins ${ }^{2}$, Yuxiang Feng ${ }^{3}$, \\ Eduardo Candela ${ }^{4}$, Marc Stettler ${ }^{5}$, Panagiotis Angeloudis ${ }^{6}$
}

\begin{abstract}
The increasing degree of connectivity between vehicles and infrastructure, and the impending deployment of autonomous vehicles (AV) in urban streets, presents unique opportunities and challenges regarding the on-street parking provision for AVs. This study develops a novel simulationoptimisation approach for intelligent curbside management, based on a metaheuristic technique. This approach is tested using an idealised grid layout with a range of flow rates and parking policies. The hybrid method balances curb lanes for driving or parking, aiming to minimise the average traffic delay. Results demonstrate delays decreased by 9\%-27\% from the benchmark case. Additionally, the delay distribution indicates the trade-offs between expanding road capacity and minimising traffic demand through curb management, demonstrating the interplay between curb parking and traffic management in the AV era.
\end{abstract}

\section{INTRODUCTION}

On-street or curbside parking, has attracted increasing interest in transport management in recent years [1], [2]. On-street parking has many benefits, compared with those off-street parking facilities. Namely, it can effectively save the scarce land resource for other development purposes, improve the utilisation of road space, alleviate congestion caused by unnecessary cruising to parking facilities, shorten walking distances, and even create barriers between heavy traffic and pedestrians [1]-[3]. However, previous studies have also indicated that on-street parking manoeuvres can block traffic, reduce road capacity, increase potential hazard among road users and increase average traffic delays [4]-[9].

There are unique opportunities and challenges with regard to on-street parking for autonomous vehicles. In the first instance, in the AVs era, off-street parking facilities will be freed up for land reclamation [10], [11], leading higher dependency on curb parking. Second, self-cruising ability enables AV parking locations no longer to be confined within a close range of destinations [2], [12], meaning parking demands can be managed with a broader geographical scope. Third, the pick-up and drop off (PUDO) feature of AVs can shorten the occupancy time of on-street parking [13], contributing to the greater flexibility of the curb lane use.

\footnotetext{
${ }^{1}$ Qiming Ye, qiming.ye18@imperial.ac.uk

${ }^{2}$ Simon M Stebbins, s.stebbins@imperial.ac.uk

${ }^{3}$ Yuxiang Feng, y.feng19@imperial.ac.uk

${ }^{4}$ Eduardo Candela, e.candela-garza19@imperial.ac.uk

${ }^{5}$ Marc Stettler, m.stettler@imperial.ac.uk

${ }^{6}$ Panagiotis Angeloudis, p.angeloudis@imperial.ac.uk

All authors are with the Centre for Transport Studies, Department of Civil and Environmental Engineering, Imperial College London, South Kensington, SW7 2AZ, London
}

Significantly, the increasing degree of connectivity between AV fleets and infrastructure will allow versatile and efficient utilisation of curbsides. To the knowledge of the authors, there are few guidelines for sizing and placing parking areas [13], [14]. Previous research on vehicle parking has not probed into these related issues.

The present study addresses these new challenges to the on-street parking problem. We conceived a simulationoptimisation framework that utlises a genetic algorithm (GA) to balance the curb use between parking and traffic circulation. The objective is to minimise the average traffic delay of AVs in the system, under different flow rates. As an essential part of the method, the traffic simulation tool SUMO (Simulation of Urban Mobility) and the metaheuristic technique (a refined genetic algorithm) have been integrated and employed. A grid network has been established, which contains sixteen $250 \mathrm{~m} \times 250 \mathrm{~m}$ urban blocks with $80 \mathrm{curb}$ lanes and 2000 parking slots. Varied combinations of parking service rates $(\alpha)$ and AV flow rates $\left(q_{\max }\right)$ have been tested.

A method is proposed to optimise the curbside use intelligently under varied parking restrictions and traffic assignment conditions. The study facilitates to combine the design of transport infrastructure (DTI) and modelling traffic assignment (TA). The application of this study is beneficial to transport and urban planning professionals, allowing them to handle the complex dynamic traffic management and infrastructure planning problem in a coordinated fashion.

\section{LITERATURE REVIEW}

Previous research concerning the on-street parking problem predominantly concentrates on three areas: the demand, supply and pricing for parking [15], [16]; safety and delay impacts of curb parking behaviours [17], [18]; the impact of on-street parking on traffic operations [19], [20]. A subset of studies from the third group has investigated how onstreet parking patterns can have an impact on such traffic operations [21]-[23]. To address this issue, these studies used cellular automata models [8], queuing models including the $\mathrm{M} / \mathrm{M} / \infty$ model [24], M/M/C model [25] and agent-based models [26] to estimate the additional travel cost and traffic congestion caused by on-street parking manoeuvres. Some studies utilised microscopic traffic simulation software, including VISSIM and SUMO (Simulation of Urban Mobility) [19], [27]. We have identified the following research gaps from the reviewed studies. 


\section{A. Modelling Objectives}

One significant gap lies in the modelling objective. A wide range of studies optimised a collection of variables other than the average traffic delay. One related work built up a network queuing model [20] to maximise the occupancy of curb parking space. A similar approach was used by Wang et al. [28], who minimised cruising distances, walking distance and coverage of amenities to balance on-street and off-street parking supply. Besides these variables, road capacity [19], [21], cruising time [29] and parking service rate [26] are all objective variables options according to previous studies. However, few studies have minimised traffic delay to obtain the optimal distribution of curb parking.

\section{B. Simplification of Traffic Operations}

Due to the complexity of interactions between on-street parking behaviours and transport operations, previous mathematical models often simplified the representation of traffic congestion, vehicular delays and microscopic driving manoeuvres [20], [21], [23], [28]. Such a simplification would lead to an approximation of the system or even inaccurate outcomes. Compared with the mathematical models, the simulation-based optimisation method can provide a finegrained and accurate representation of discrete traffic units [29] and simulates complex manoeuvres.

Studies adopting the simulation methods can directly model the complicated operations of traffic flow and parking manoeuvres [19], [25], [27], [29]. For instance, Polycarpou et al. [15] deployed a SUMO-based optimisation model to find the optimal matching solutions between parking slots and drivers [29]. The significant advantage of the simulationbased approach lay in the fact that it can provide a finegrained and accurate representation of microscopic traffic operations. However, this method did not seek to optimise the distribution of parking space, nor to minimise the average delay. Neither did it manage the curb lane use dynamically through recursively running the traffic simulation.

\section{Generality of Models}

Most case-based studies only investigate the issue based on a specific flow pattern or a fixed parking pattern. Their findings are likely to be profoundly affected by factors that are specific to the case study, such as the geometry of road grids, signal timing, parking space geometries and interference from other active modes of transport [5], [6], [27], [30]. To alleviate the impact of these specific factors, it is critical to develop an ideal and generic environment which simulates the movements of AVs. Besides, testing varied parking policies under different traffic saturation conditions is equally significant.

\section{METHODOLOGY}

We establish a simulation-based genetic algorithm to optimise the curb lane use, minimising average traffic delay of AVs. This method finds an optimal on-street parking pattern under varying flow rates and parking service rates. Two essential parts comprise the main body of our method:

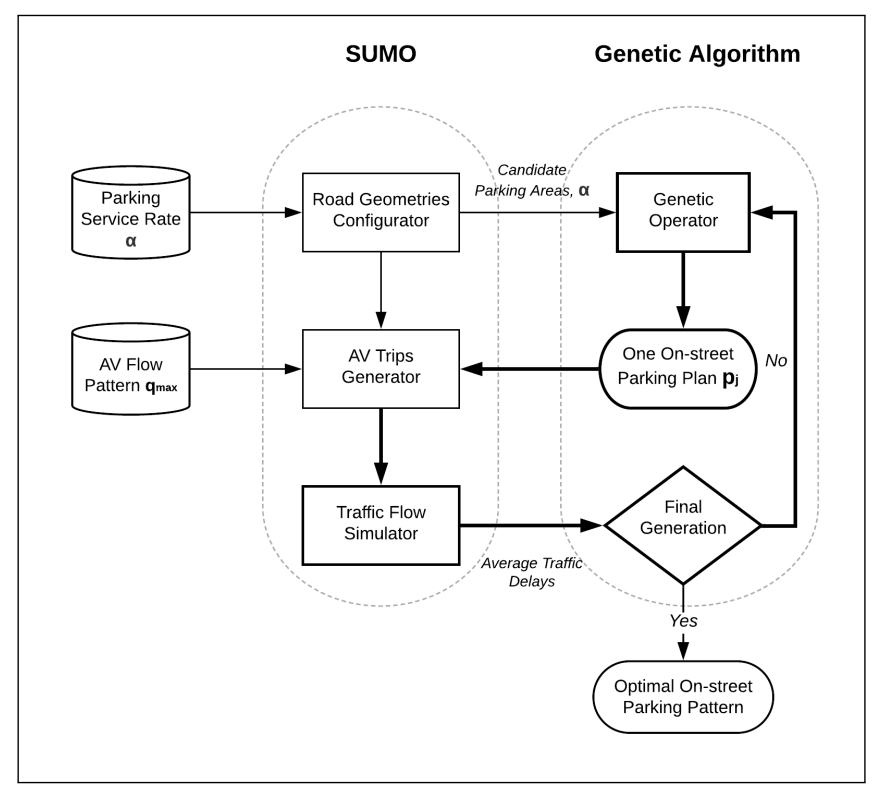

Fig. 1. Flow Chart of On-street Parking Areas Optimisation Model

SUMO (Simulation of Urban Mobility) evaluates the plans proposed by a genetic algorithm (GA).

Respectively, the primary function of SUMO is to configure the geometries of road grids and curb parking areas, to generate vehicular routes, and to simulate traffic flow. On top of that, a GA is employed and integrated with SUMO to optimise the curb parking plan $(p)$. The reason for choosing such a metaheuristic technique is that it has shown high suitability and efficiency in solving complex optimisation problems [26], [29]. Fig.1 illustrates the simulation and optimisation workflow between the two parts, with bold arrows highlighting the optimisation process using the GA.

\section{A. Configuration Environment}

A geometry configurator creates a grid plan consists of 16 urban blocks as a virtual simulation environment. Sixty 250 meter-long bi-directional streets comprise the basic frame of the network, in which the central forty streets are embedded with curb lanes prepared for parking. The twenty remaining peripheral streets are used as guiding paths for AVs to enter and leave the system. For each link direction, there is a curbside and an overtaking lane. At each end of a curb lane, adequate space remains to avoid the bottleneck effect [23], leaving the maximum capacity of 25 available slots for each curb lane. Fig.2 demonstrates this simulation environment with detailed streets layout at an intersection.

Routes are generated based on the network geometry, flow rates, parking locations and parking policy. For all routes from entrance $a$ to exit $b$, where $(a, b) \in O D s$ (the set of all origin-destination pairs), flow $f_{a, b}$ is equal to the sum of all vehicular trips from $a$ to $b$. Note that there are multiple possible routes for a single trip $r$, depending on which intersections a vehicle passes through, where $r \in R_{a, b}$ is one such route that originates at $a$ and finishes at $b$. The value of $f_{a, b}$ follows a uniform distribution given the maximum flow 


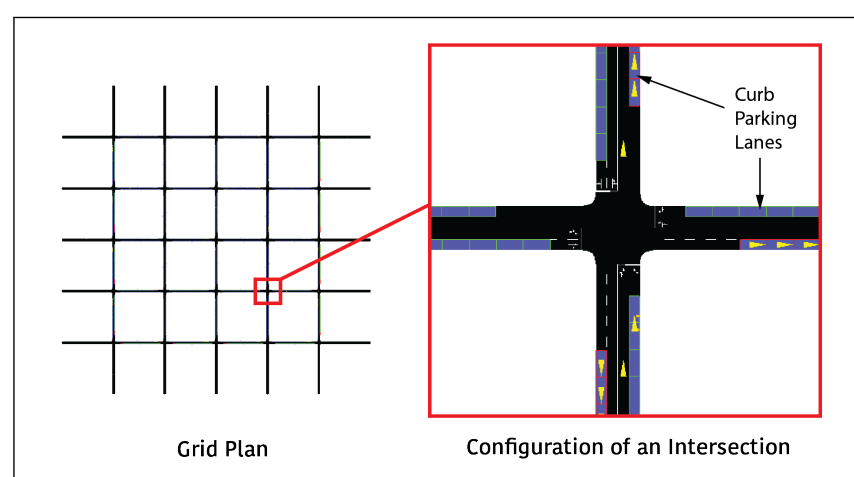

Fig. 2. SUMO Configuration of the Road Network

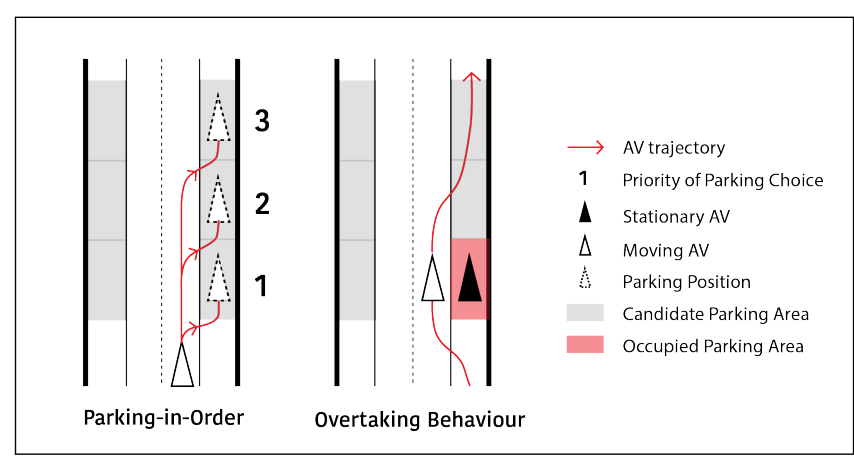

Fig. 3. Behaviour 1: Overtaking A Stationary AVs

rate $q_{\max }$, which is expressed in Eq.1.

$$
f_{a, b}=\sum_{r \in R_{a, b}} f_{r}=\operatorname{rand}\left(0, q_{\max }\right] \quad \forall a, b \in O D s
$$

Two important vehicular operations are realised to manage the use of curb parking intelligently. As Fig. 3 shows, the first operation is parking-in-order, which requires vehicles to primarily occupy vacant slots closest to the upstream entry of a lane. It means that the front unoccupied curb spaces can still be used for circulating traffic flow. On top of that, the second essential operation is to permit successive vehicles to overtake a stationary vehicle. In this fashion, on-street parking only occupies reasonable space instead of blocking the whole road, thereby reducing capacity without cutting the flow of vehicles entirely. However, parked vehicles will not participate in traffic circulation, reducing traffic demand of the system. Therefore, allocating curb lanes for parking introduces a trade-off between road capacity and traffic demand.

\section{B. Optimisation with Genetic Algorithm}

Genetic algorithms (GAs) are a class of metaheuristic algorithms that are used to find high-quality solutions to largescale, non-linear optimisation problems [31]. Inspired by genetic processes in biology, GAs apply selection, crossover and mutation operations to imitate the natural selection process [32]. The search spaces are often enormous. The number of possible solutions to the parking space allocation problem is $\left(\begin{array}{l}n_{c} \\ n_{p}\end{array}\right)$, where $n_{c}, n_{p}$ denote the number of candidate
TABLE I

VARIABLES AND PARAMETERS FOR SUMO-GA

\begin{tabular}{lc|ccccc}
\hline Parameters & Symbol & \multicolumn{5}{c}{ Values } \\
\hline Max Generations & $I$ & 100 & & & & \\
Population Size & $s$ & 12 & & & & \\
Selection Rate & $\eta$ & 0.5 & & & & \\
Parking Service Rate & $\alpha$ & 0 & 0.25 & 0.50 & 0.75 & 1 \\
Parking Candidates & $n_{c}$ & 80 & & & & \\
Parking Areas & $n_{p}$ & 0 & 20 & 40 & 60 & 80 \\
Max flow & $q_{\max }$ & 10 & 25 & 40 & 55 & 70 \\
\hline
\end{tabular}

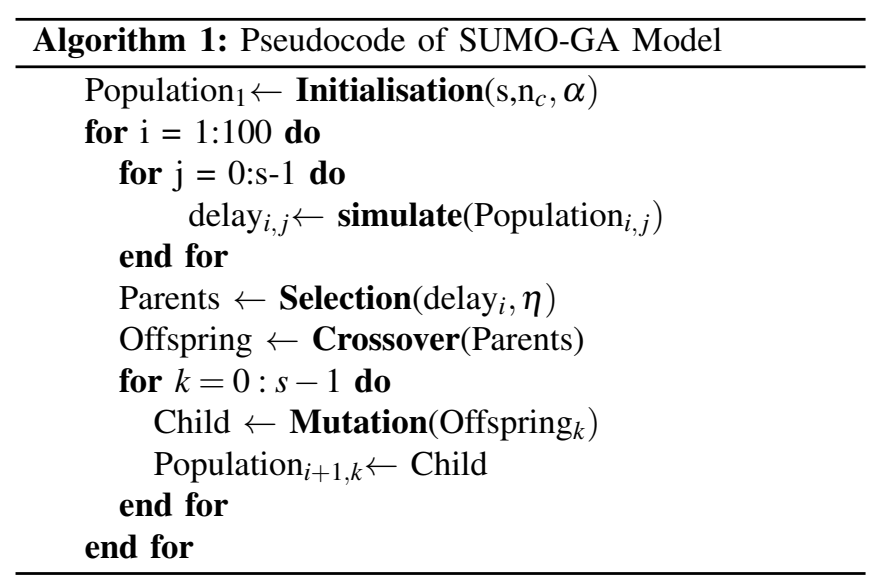

parking areas and the number of permitted parking areas respectively. For instance, with a parking service rate of 0.5 , and 80 candidate parking areas, the total number of possible solutions is $\frac{80 !}{40 ! 40 !} \approx 10^{23}$. Despite the magnitude of the search space, the GA quickly converges to a locally optimal solution.

The optimisation problem is represented as follows. Given the AV flow patterns $q$, the model tries to find the optimal pattern of on-street parking distribution $p$, which has the highest fitness score, namely the lowest average traffic delay D. As expressed in Formula.2 and Eq.3, the objective is to minimise $D$ over the control $p$, and input $q . V$ is the set of vehicle indices. $T_{i}$ is travel time calculated for vehicle $i$, from the time the AV enters into the network until it leaves. Given $q, F_{i}$ represents the delay-free time required for vehicle $i$ to pass through the network. A parked AV stays in a curb parking space for half an hour, and this time does not contribute toward delay.

$$
\begin{array}{ll}
\text { minimise } & D(p ; q) \\
& D(p ; q)=\frac{1}{|V|} \sum_{i \in V}\left(T_{i}(p ; q)-F_{i}(x)\right)
\end{array}
$$

The GA method consists of five essential steps: population initialisation, traffic delay simulation, parents selection, offspring crossover and mutation. Algorithm.1 outlines the optimisation process and Table.I describes parameters and variables used in this section.

Initialisation The initial step is to create the generation zero population, as demonstrated in Fig.4. Binary values $(0,1)$, as the basic genes, represent the serving states of 80 


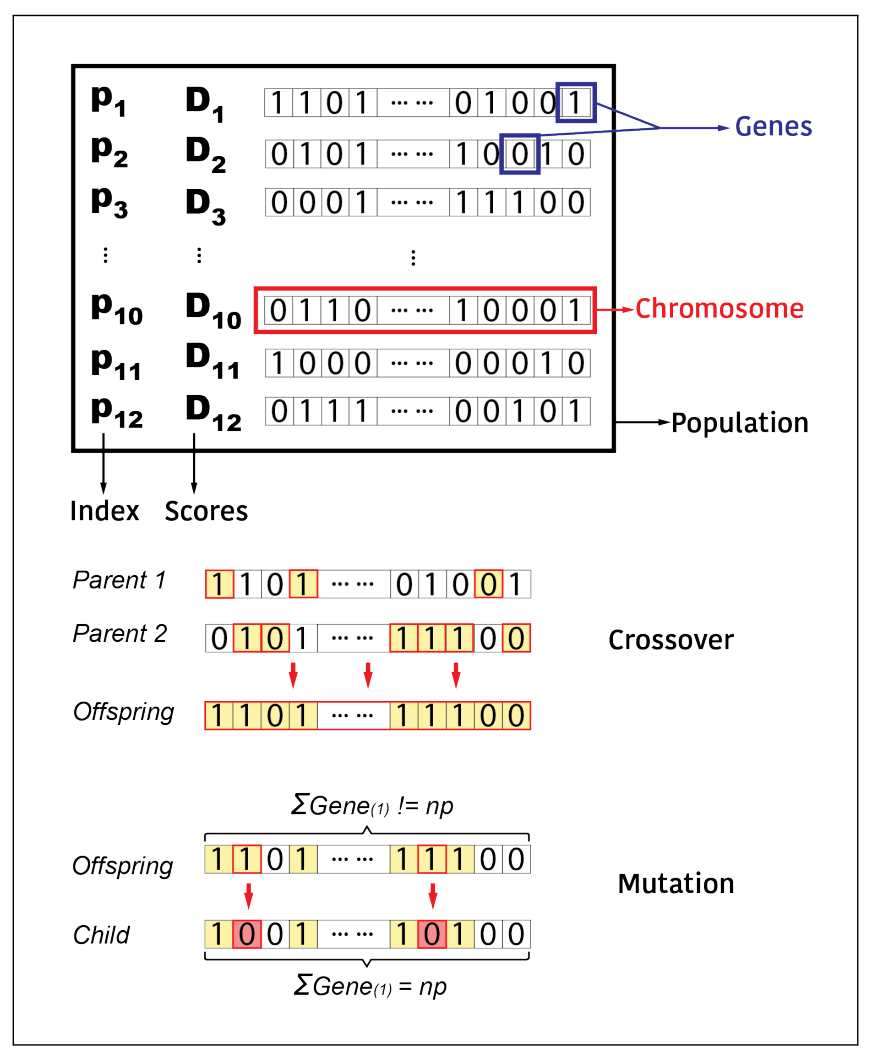

Fig. 4. Illustration of Population, Crossover and Mutation of GA

parking areas. 1 indicates an available parking area and 0 indicates an area where parking is currently prohibited. The total permitted number of $1 \mathrm{~s}$, is determined by the number of candidate parking areas and the parking service rate $\alpha$, following a function expressed in Eq.4. Genes then form a binary sequence, known as a chromosome, representing a possible curb parking distribution plan $p$. Generally, $s$ chromosomes construct a population, which is the fundamental unit to evolve through $i$ generations.

Calculation of Traffic Delay Each chromosome, as a plan $\left(p_{j}\right)$, can now be used to allocate an available parking area. SUMO then simulates traffic and calculates the average delay $D_{j}$ of the plan $p_{j}$. With these computed delays (one for each chromosome), the method selects elite chromosomes following a selection strategy in Eq.5 with rate $\eta$. Only the elite chromosomes, called parents, are eligible for mating in the next step. We set selection rate $\eta=0.5$ here, indicating that only six parents (Parents $s_{i}$ ) out of the 12 chromosomes are allowed to evolve for each generation.

$$
\begin{aligned}
n_{p} & =\alpha \cdot n_{c} \\
n_{\text {parents }} & =\eta \cdot s
\end{aligned}
$$

Crossover and Mutation The final step is to generate twelve children out of the elite parents, through crossover and mutation procedures. The crossover process shuffles genes of a pair of parents to produce four offspring units randomly. The offspring units are the 'premature' in the sense that they might have excessive one or zero genes. An excess of one or zero genes violates the parking policy, which should be prohibited. To avoid this, a mutation operator randomly removes the excessive genes to achieve the parking service rate $(\alpha)$. The children, as qualified chromosomes, eventually form a new generation of the population. The GA optimisation process continues until all chromosomes in a generation converge to the same solution. In all tests, convergence occurred in less than 100 generations.

\section{RESULTS AND DISCUSSION}

In this section, we first analysed the optimal curb parking results on the basis of two scenarios. Followed by that, a convergence performance diagram has been attached to demonstrate the optimisation ability of this designed SUMOGA method. We further investigated the distribution of average delay of all scenarios, for both the base circumstance and the GA-optimised condition. Additionally, the utility of this method has been discussed by comparing the improvements of average delay across scenarios.

\section{A. Optimal Patterns of Curb Parking}

Testing was conducted for twenty-five scenarios based on a variety of parking provision rates and maximum flows. The parking service rates tested were $(\alpha=0,0.25,0.5,0.75$ and 1) and maximum flows generated for each OD pair were $\left(q_{\max }=10,25,40,55\right.$ and $\left.70 \mathrm{veh} / \mathrm{h}\right)$. Results based on all scenarios demonstrated a good optimisation ability of this SUMO-GA method. In general, the method reduced the average traffic delay by $7 \mathrm{~s}-57 \mathrm{~s}$, which corresponds to a relative decrease of $9 \%-27 \%$ compared to the average result of generation 0 of the GA.

Fig.5 demonstrates the optimised distribution of curb parking lanes in two scenarios, namely, $\alpha=0.25$ with $q_{\max }=10$, and $\alpha=0.5$ with $q_{\max }=70$. The colours represent the use of curb lanes, with red for parking and grey for driving. Line width and opacity indicate the magnitude of traffic flow assigned to each link. It can be noted that, when both parking service rate and traffic flow rate are low, curb parking areas are likely to be allocated to avoid links with heavy traffic. As the two variables increase, such spatial division becomes less visually obvious.

Fig. 6 shows the convergence of the GA under the scenario of $\alpha=0.5$ and $q_{\max }=70 \mathrm{veh} / \mathrm{h}$. The optimisation converges at generation 33 with an average delay of 490s. Even though the delay does not converge monotonically, due to the stochastic nature of metaheuristic methods, such declining trend is quite clear. Similarly, in other scenarios, the average delays generally converged around $32( \pm 15)$ generations, meaning all tests converge far earlier than the maximum 100 generations.

\section{B. Traffic Saturation Thresholds}

The average traffic delays were acquired of all 25 scenarios with different max flow rates $\left(q_{\max }=10,25,40,55,70 \mathrm{veh} / \mathrm{h}\right)$ and on-street parking service rates $(\alpha=0,0.25,0.5,0.75$ and 1) using SUMO. The average delay before GA optimisation is denoted as $D_{0}$ for the benchmark circumstance, which 


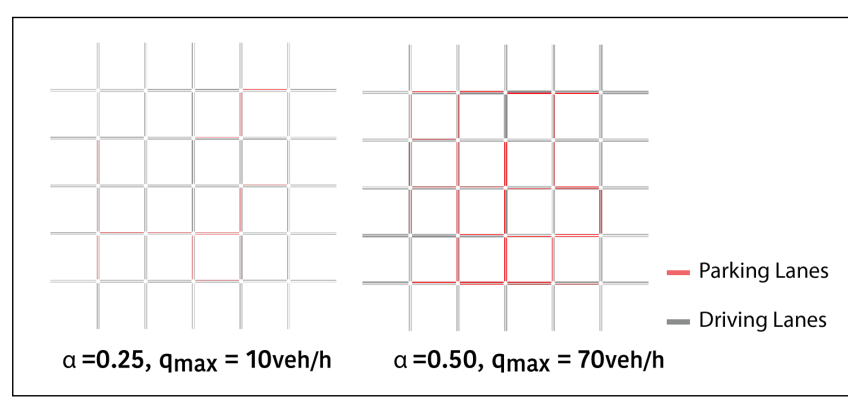

Fig. 5. Optimal Curb Parking Distribution Under Two Scenarios

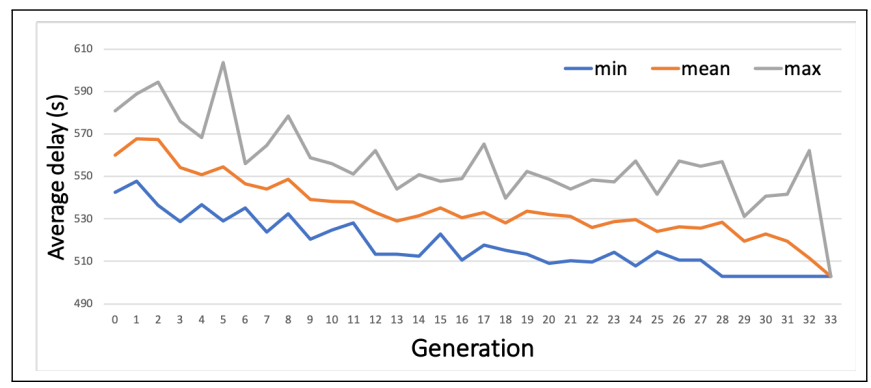

Fig. 6. Convergence of Genetic Algorithm Over Successive Generations

equals the average delay of generation $0 . D^{*}$ denotes the optimised average delay after GA optimisation.

Two curved surfaces in Fig.7 demonstrate the distribution of the average delay of $D_{0}$ and $D^{*}$. When $\alpha$ is constant, the average delay increases drastically as the flow rate increases. This indicates that traffic delay is predominantly determined by the traffic flow rather than parking service rate. This is further evidenced by comparing the average coefficient of variations $(C V)$ while varying two variables. When flow rate varies, $C V=1.08(>0.35)$, suggesting a relatively high degree of variation. In comparison, when the parking service rate varies, changes in delay are not significant, with $C V=0.24$.

The gradients in both figures display prompt and steep increases at the max flow rate of 40-55 veh $/ h$ with an average gradient of $24.87 \mathrm{~s} / \mathrm{veh} \cdot h^{-1}$. Compared with $0.83 \mathrm{~s} / \mathrm{veh} \cdot \mathrm{h}^{-1}$ with lower flow rates, the sharp rise implies the existence of a threshold between the under-saturated and over-saturated traffic. As traffic builds up, the congestion level is likely to increase in a near exponential fashion [33].

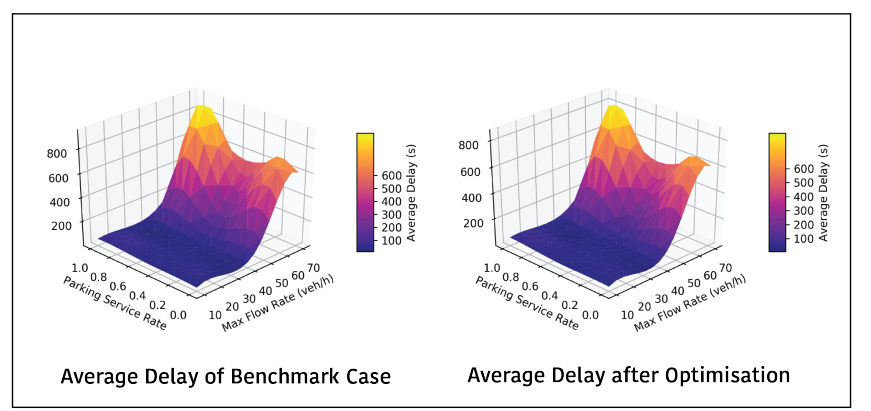

Fig. 7. Initial and SUMO-GA Optimised Traffic Delay

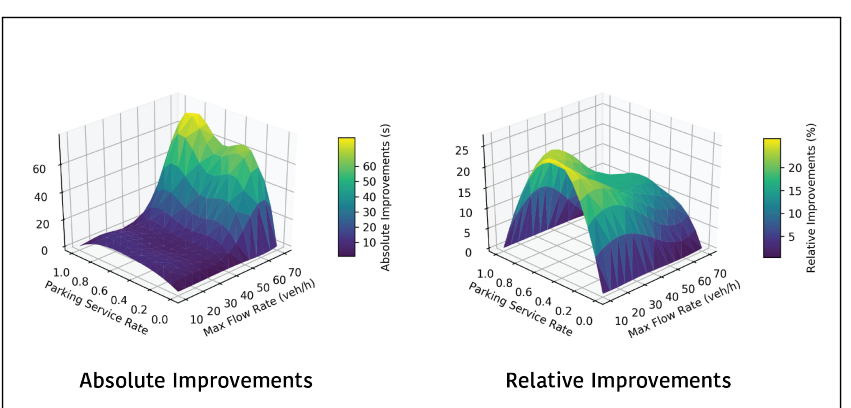

Fig. 8. Absolute and Relative Improvements of Delay

\section{Trade-offs Between Driving and Parking}

Two concave regions appear in both curved surfaces around $\alpha=0.5, q_{\max }=55-70 \mathrm{veh} / h$. These concave regions represent the trade-offs between reducing road capacity and reducing traffic demand. Concretely, in saturated traffic scenarios, if half of the curb lanes are utilised for parking, the average delay significantly reduces from $416 \mathrm{~s}(\alpha=1)$ and $494 \mathrm{~s}(\alpha=0)$ to $346 \mathrm{~s}$. These trade-offs highlight the fact that even though the traffic is over-saturated, the average delay is reduced by temporarily occupying driving lanes for parking.

The rationale of this compromise in over-saturated traffic conditions can be explained by investigating the relationship between traffic demand and road capacity. Given a fixed flow rate, when parking is prohibited $(\alpha=0)$, curb lanes primarily serve traffic flow. By restricting curb lane use to driving, traffic congestion is expected to alleviate, and average delay declines due to an increase in capacity. However, our results provide evidence that curb parking may also contribute to reducing delays, as vehicles are removed from the driving lanes and this restrains traffic demand. In other words, implementing appropriate parking policies can benefit traffic operations. Thus, our results indicate a complex correlation for this on-street parking problem. These findings extend previous studies [19], [21], which attempted to prove a negatively correlated, linear relationship between on-street parking and transport efficiency.

\section{Map of Improvements}

Fig.7 demonstrates the absolute improvement of average delay (left figure), and the relative improvement (right figure) respectively. The absolute improvement, equivalent to $D_{0}-D^{*}$, echos the trends shown in the previous Fig.8. The function plotted in figure 7 indicates a maximum difference of $55.60 \mathrm{~s}$ per $\mathrm{AV}$ at the max flow rate of $70 \mathrm{veh} / \mathrm{h}$. The results show that the method is more effective at reducing average delay in over-saturated traffic conditions.

On the other hand, the relative improvements figure (Fig. 8) demonstrates how much delay has been reduced in comparison with the benchmark circumstance, namely $\left(D_{0}-\right.$ $\left.D^{*}\right) / D_{0}$. The saddle-like distribution of these improvements shows that relative performance is better for under-saturated traffic conditions. An AV saves, on average, $28 \%$ delay with the implementation of this method. The high relative reduction at the parking service rate of 0.5 suggests it is the 
optimal parking policy for the scenarios tested. This finding reflects the previous conclusions made about the trade-offs between driving and parking.

\section{CONCLUSIONS}

This study provides a solution for the the curb lane parking provision problem, taking into consideration the unique aspects that AVs introduce. A SUMO simulationbased genetic algorithm has been deployed to optimise the curb lane use in accordance with traffic flow conditions and parking policies. A virtual grid plan and AV flows were configured in SUMO, and the curb lane parking plans were represented and optimised using a GA. Twenty-five scenarios with varied max flow rates and differing parking service rates were investigated.

The results demonstrate a trade-off between traffic demand and network capacity, as the parking provision service rate changes. Additionally, the concave region of the delay distribution shows that curb parking management effectively optimises reductions in traffic delay, even for over-saturated conditions. It was demonstrated that the proposed method is capable of lowering average delay in both under- and over-saturated cases. However, it is more beneficial in oversaturated cases.

This study provides a new scope to solve the combined problem of dynamic traffic assignment and transport infrastructure design. Further work will focus on improving the robustness of the method by introducing more computationally efficient, macroscopic models. It would also be beneficial to investigate the application of this method to street design and traffic management in a real-world setting.

\section{REFERENCES}

[1] G. Marsden, "The evidence base for parking policies-a review," Transport policy, vol. 13, no. 6, pp. 447-457, 2006.

[2] A. Millard-Ball, "The autonomous vehicle parking problem," Transport Policy, vol. 75, pp. 99-108, 2019.

[3] S. Biswas, S. Chandra, and I. Ghosh, "Effects of on-street parking in urban context: A critical review," Transportation in developing economies, vol. 3, no. 1, p. 10, 2017.

[4] A. Ramadan and M. J. Roorda, "An integrated traffic microsimulation model of illegal on-street parking in downtown toronto," in The 96th international conference of Transportation Research Board, 2017, pp. $17-05970$.

[5] S. Wijayaratna, "Impacts of on-street parking on road capacity," in Australasian Transport Research Forum, 2015, pp. 1-15.

[6] H. Guo, Z. Gao, X. Yang, X. Zhao, and W. Wang, "Modeling travel time under the influence of on-street parking," Journal of Transportation Engineering, vol. 138, no. 2, pp. 229-235, 2012.

[7] A. I. Portilla, B. A. Oreña, J. L. Berodia, and F. J. Díaz, "Using $\mathrm{m} / \mathrm{m} /$ queueing model in on-street parking maneuvers," Journal of Transportation Engineering, vol. 135, no. 8, pp. 527-535, 2009.

[8] G. Jingqin and O. Kaan, "Modeling double parking impacts on urban street," in 95th Annual Meeting of the Transportation Research Board (TRB), 2016

[9] P. C. Box, "Curb-parking problems: Overview," Journal of Transportation Engineering, vol. 130, no. 1, pp. 1-5, 2004.

[10] A. Noyman, A. Stibe, and K. Larson, "Roadmap for autonomous cities: Sustainable transformation of urban spaces," 2017.

[11] W. Zhang and S. Guhathakurta, "Parking spaces in the age of shared autonomous vehicles: How much parking will we need and where?" Transportation Research Record, vol. 2651, no. 1, pp. 80-91, 2017.

[12] D. Stead and B. Vaddadi, "Automated vehicles and how they may affect urban form: A review of recent scenario studies," Cities, vol. 92, pp. 125-133, 2019.
[13] NACTO, "Blueprint for autonomous urbanism," 2017, [Online; accessed 07-Apr-2019]. [Online]. Available: https://nacto.org/publication/bau/blueprint-for-autonomous-urbanism/

[14] A. Noyman, A. Stibe, and K. Larson, "Autonomous cities and the urbanism of the 4th machine age: Should av industry design future cities," Changing Places Research Group, MIT Media Lab, accessed March, vol. 29, 2016.

[15] E. Polycarpou, L. Lambrinos, and E. Protopapadakis, "Smart parking solutions for urban areas," in 2013 IEEE 14th International Symposium on" A World of Wireless, Mobile and Multimedia Networks"(WoWMoM). IEEE, 2013, pp. 1-6.

[16] O. Cats, C. Zhang, and A. Nissan, "Empirical evaluation of an on-street parking pricing scheme in the city center," in 94th Annual Meeting of the Transportation Research, vol. 29, 2015.

[17] K. Małecki, "A computer simulation of traffic flow with on-street parking and drivers' behaviour based on cellular automata and a multiagent system," Journal of computational science, vol. 28, pp. 32-42, 2018.

[18] A. Naseri, "A method for estimating average delay time due on-street parking," Australian Journal of Basic and Applied Sciences, vol. 7, no. 8, pp. 767-774, 2013.

[19] C. Gkini, C. Iliopoulou, K. Kepaptsoglou, and E. I. Vlahogianni, "Model for planning and sizing curbside parking lanes in urban networks," Transportation Research Record, vol. 2672, no. 20, pp. $1-11,2018$.

[20] C. P. Dowling, L. J. Ratliff, and B. Zhang, "Modeling curbside parking as a network of finite capacity queues," IEEE Transactions on Intelligent Transportation Systems, 2019.

[21] C. Mutiawati, L. Lulusi, F. M. Suryani, and S. Sugiarto, "Feasibility of on-street parking based on degree of saturation," Aceh International Journal of Science and Technology, vol. 7, no. 1, pp. 11-23, 2018.

[22] S. Yousif and Purnawan, "Traffic operations at on-street parking facilities," in Proceedings of the Institution of Civil Engineers-Transport, vol. 157, no. 3. Thomas Telford Ltd, 2004, pp. 189-194.

[23] J. Cao, M. Menendez, and V. Nikias, "The effects of on-street parking on the service rate of nearby intersections," Journal of Advanced transportation, vol. 50, no. 4, pp. 406-420, 2016.

[24] H. Guo, W. Wang, and W. Guo, "Micro-simulation study on the effect of on-street parking on vehicular flow," in 2012 15th International IEEE Conference on Intelligent Transportation Systems. IEEE, 2012, pp. $1840-1845$.

[25] Y. Guo, Y. Zhang, Y. Zhao, Q. Jia, Z. Jing, Y. Ren, R. Li, and $\mathrm{X}$. Luo, "Traffic congestion evaluation and management optimization based on queueing model and vissim simulation," in 2018 IEEE 22nd International Conference on Computer Supported Cooperative Work in Design ((CSCWD)). IEEE, 2018, pp. 672-676.

[26] Z. Mei, W. Zhang, L. Zhang, and D. Wang, "Optimization of reservation parking space configurations in city centers through an agentbased simulation," Simulation Modelling Practice and Theory, vol. 99, p. 102020,2020 .

[27] T. Limanoond et al., "Impact of on-street parking on urban arterial performance: A quantitative study on travel speed and capacity deterioration," Aceh International Journal of Science and Technology, vol. 2, no. 2, pp. 63-69, 2013.

[28] S. WANG, J. CHEN, and H. ZHANG, "A location optimization model for curb parking facilities coordinating with off-street parking facilities," Journal of Highway and Transportation Research and Development, vol. 5, 2009.

[29] J. Arellano-Verdejo and E. Alba, "Optimal allocation of public parking slots using evolutionary algorithms," in 2016 International Conference on Intelligent Networking and Collaborative Systems (INCOS). IEEE, 2016, pp. 222-228.

[30] J. Chen, Z. Li, W. Wang, and H. Jiang, "Evaluating bicycle-vehicle conflicts and delays on urban streets with bike lane and on-street parking," Transportation letters, vol. 10, no. 1, pp. 1-11, 2018.

[31] X.-S. Yang, "Review of metaheuristics and generalized evolutionary walk algorithm," arXiv preprint arXiv:1105.3668, 2011.

[32] K. A. De Jong, "Analysis of the behavior of a class of genetic adaptive systems," Tech. Rep., 1975.

[33] S. Stebbins, M. Hickman, J. Kim, and H. L. Vu, "Characterising green light optimal speed advisory trajectories for platoon-based optimisation," Transportation Research Part C: Emerging Technologies, vol. 82, pp. 43-62, 2017. 